\title{
Tuning quantum correlations with intracavity photonic crystals
}

\author{
Maria M. de Castro, ${ }^{1}$ Miguel Angel García-March, ${ }^{2}$ Damia Gomila, ${ }^{1}$ and Roberta Zambrini ${ }^{1}$ \\ ${ }^{1}$ IFISC, Institute for Cross-Disciplinary Physics and Complex Systems (CSIC-UIB), Campus UIB, E-07122 Palma de Mallorca, Spain \\ ${ }^{2}$ Department of Physics, Colorado School of Mines, Golden, Colorado 80401, USA \\ (Received 3 November 2010; revised manuscript received 31 March 2011; published 19 September 2011)
}

\begin{abstract}
We show how to tune quantum noise in nonlinear systems by means of periodic spatial modulation. We prove that the introduction of an intracavity photonic crystal in a multimode optical parametric oscillator inhibits and enhances light quantum fluctuations. Furthermore, it leads to a significant noise reduction in field quadratures, robustness of squeezing in a wider angular range, and spatial entanglement. These results have potential benefits for quantum imaging, metrology, and quantum information applications and suggest a control mechanism of fluctuations by spatial modulation of interest also in other nonlinear systems.
\end{abstract}

DOI: $10.1103 /$ PhysRevA.84.033832

PACS number(s): 42.65.Yj, 42.50.Dv, 42.70.Qs

\section{INTRODUCTION}

Photonic crystals (PCs) are dielectric media with a periodic modulation of the refractive index that can lead to gaps in the allowed frequencies of electromagnetic waves, which are analogous to electronic band gaps in semiconductor devices $[1,2]$. Inside a PC, a radiative transition at some frequency $\omega$ can be suppressed when it falls within the band gap [1]. The seminal idea about the possibility of controlling spontaneous emission by PCs [1] has recently been demonstrated experimentally [3], improving the extraction efficiency of lightemitting devices and redistributing the corresponding energy where needed [4]. The use of PCs for environment (dissipation) engineering is also the basis of intense research activity about the non-Markovian evolution of quantum states [5]. These engineered media are a versatile tool to control light both at the level of spontaneous emission and for intense optical fields, providing an unprecedented control of light confinement, guiding, and propagation [2,6]. PC frequency band gaps have been proposed also to inhibit the excitation of high-order transverse modes in nonlinear cavities [7]. In this case, the emission of tilted light beams above some threshold (modulation instability) is forbidden if it falls within the band gap of an intracavity PC, as recently observed in two independent experiments [8].

The possibility of accessing the information encoded in different spatial modes in nonlinear devices has been at the origin of intense research in multimode quantum optics [9]. This effort has culminated in several applications recently demonstrated, such as optical switching [10], quantum imaging [11], metrology [12], and quantum information [13]. In particular, quantum correlated bright beams can be generated by optical parametric oscillators (OPOs), which are common nonlinear devices for frequency down-conversion and for squeezing [14] and entanglement [15] between light modes with different polarizations, frequencies, or wave vectors [16-18]. In this context the use of PCs to control quantum correlations has not been considered, although it could be a key tool for improving performance in practical applications.

In this paper we show how to control and improve multimode squeezing and entanglement by means of an intracavity PC in a nonlinear device. The fundamental question we address is whether a spatial modulation in a nonlinear system allows one to reduce quantum fluctuations and to improve quantum correlations. As a prototypical system we consider a multimode degenerate OPO $[17,18]$ considering quantum fluctuations changes when a PC is introduced in the cavity. In the proposed photonic-crystal optical parametric oscillator (PCOPO), the spatial modulation is not changing the environment's spectrum of fluctuations or confining light, but is instead modifying the intracavity process. The PC modulation takes place in the transverse plane with respect to the propagation direction of both pump and down-converted light beams and is modeled by a spatial profile of the otherwise homogeneous refractive index. We consider a doubly resonant planar ring cavity in which a quadratic medium and photonic crystal are placed, as sketched in Fig. 1. For a longitudinally monomode device and in the mean-field approximation [19], the relative positions of the quadratic crystal and PC are not relevant. For down-conversion in the type-I degenerate configuration, the generated signal has half of the frequency of the linearly polarized pump, with the orthogonal polarization. For planar mirrors this device is transversally degenerate [9,16-19]. Stripe patterns on the order of $10 \mu \mathrm{m}$ have been reported in OPOs [20]. We will consider here a refractive index modulation in the PCOPO with periodicity on the same order as the spontaneous pattern, which does not represent a special challenge with respect to the case of standard OPO devices.

After introducing the mathematical model (Sec. II), we will show that the PC allows for a large tunability of the modulation instability and parametric threshold that we explain by considering the underlying nonlinear wave mixing (Sec. III). The mean number of photons, related to light fluctuations in the PCOPO, is connected to the instability process and it can indeed be lowered or enhanced just by a change in the PC modulation amplitude. In Sec. IV quantum correlations are characterized in different regimes and two major effects are identified above threshold, namely, a substantial widening of the range of squeezed quadratures and spatial entanglement by considering either state inseparability [21] or the Einstein-Podolsky-Rosen [22] criterion of Ref. [23].

\section{PHOTONIC-CRYSTAL OPO EQUATIONS}

Quantum fluctuations in a PCOPO can be modeled with nonlinear stochastic equations by phase-space methods. The 


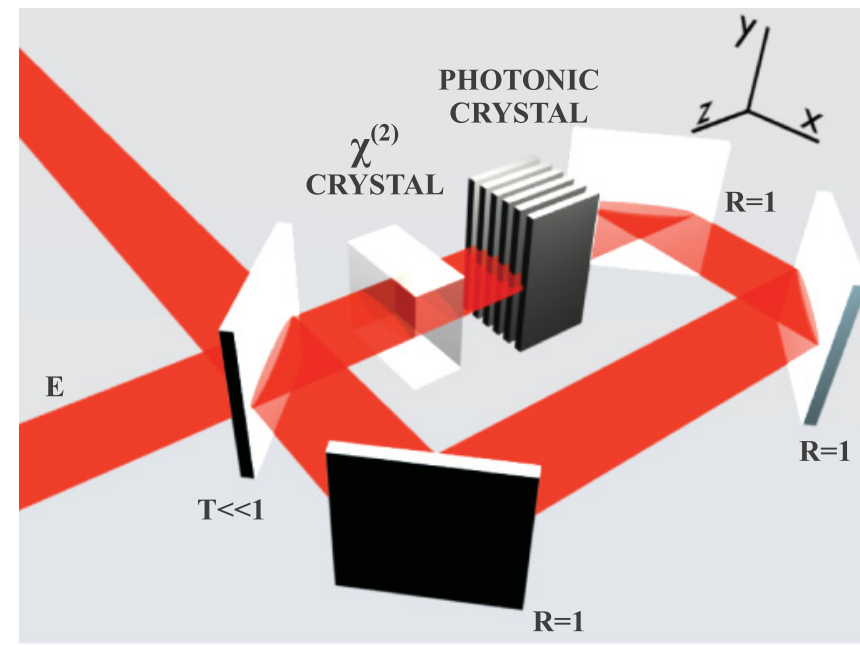

FIG. 1. (Color online) Optical parametric oscillator with a photonic crystal modulated in the transverse direction $x$. We consider a ring planar cavity, with one input mirror with a small transmission coefficient $T$, while the other mirrors have $100 \%$ reflectivity $R$ at the field's frequencies. The doubly resonant cavity contains a slice of a nonlinear medium, a $\chi^{(2)}$ crystal, and an intracavity photonic crystal with a refractive index $n=n(x)$. A coherent, stationary, plane-wave laser beam $E$ is injected into the cavity in the $z$ direction.

master equation for multimode type-I degenerate OPOs [17] can be mapped into the $\mathrm{Q}$ representation as discussed in Ref. [18]. This leads to nonlinear Langevin equations for the pump $\alpha_{0}(x, t)$ and (down-converted) signal $\alpha_{1}(x, t)$ light fields, which are valid both below and above threshold (if $\left|\alpha_{0}\right|<2$ ):

$$
\begin{aligned}
\partial_{t} \alpha_{0}(x, t)= & -\left\{\left[1+i \Delta_{0}(x)\right]-i \nabla^{2}\right\} \alpha_{0}(x, t) \\
& +E-\frac{1}{2} \alpha_{1}^{2}(x, t)+\xi_{0}(x, t), \\
\partial_{t} \alpha_{1}(x, t)= & -\left\{\left[1+i \Delta_{1}(x)\right]-2 i \nabla^{2}\right\} \alpha_{1}(x, t) \\
& +\alpha_{0}(x, t) \alpha_{1}^{*}(x, t)+\xi_{1}(x, t) .
\end{aligned}
$$

As usual, these equations describe the slowly varying field envelopes in the mean-field approximation [17,19] with $x$ the transverse direction (in one dimension) and $\nabla^{2}$ the Laplacian modeling diffraction. In the thin (nonlinear and photonic) crystal limit, deviations from free-space diffraction can be neglected [9,16-19]. Here $E$ is the homogeneous input field at the pump frequency and $\xi_{0}(x, t)$ and $\xi_{1}(x, t)$ are, respectively, additive and phase-sensitive multiplicative white noises [18,24]. The derivation of these stochastic nonlinear partial derivative equations for the OPO is given in Ref. [18] together with the scalings used and the expression of noises $\xi_{0,1}$.

The PC refractive index modulation leads to spatialdependent detunings $\Delta_{0}(x)$ and $\Delta_{1}(x)$, which can have different amplitudes and, in the simplest case, have the same periodicity with wave number $k_{\mathrm{PC}}$. In particular, the OPO homogeneous detunings $\delta_{0,1}$ become, in a PCOPO,

$$
\Delta_{0}(x)=\delta_{0}+M_{0} \sin \left(k_{\mathrm{PC}} x\right)
$$

in the pump field equation and

$$
\Delta_{1}(x)=\delta_{1}+M_{1} \sin \left(k_{\mathrm{PC}} x\right)
$$

in the signal field equation. We stress that the PC is modulated only in the transverse direction $x$ as we aim to explore the effects on the field spatial instability. In other words, the model we consider does not differ from an OPO in the propagation direction $[17,18]$ because the medium is actually homogeneous in this direction and the field does not experience any band gap. The main mechanism we explore is the effect of the transverse band gap on the spatially multimode down-conversion process. We then consider a negative $\delta_{1}=-1$ (homogeneous part of the detuning) that in an OPO would lead to a modulation instability at wave number $k_{c}=\sqrt{-\delta_{1} / 2}=\sqrt{0.5}$ [25]. Therefore, the most interesting configuration is for a PC (sinusoidal) modulation with $k_{\mathrm{PC}}=$ $2 k_{c}$ since in this case the OPO emission would be in the photonic band gap [7].

The results in the following sections are obtained by numerical simulation of the stochastic equations (2) and (3), as described in detail in Ref. [24]. We consider several configurations and present results for the OPO in the absence of PCs $\left(M_{0}=M_{1}=0\right)$, for a PCOPO with PCs affecting the signal field $\left(M_{0}=0\right.$ and $\left.M_{1}=0.5\right)$, and for a PCOPO with PCs affecting the pump field $\left(M_{0}=0.5\right.$ and $\left.M_{1}=0\right)$. The possibility of having different refractive index modulations for the pump and signal fields is physically allowed by the difference between their frequencies.

\section{TUNING SIGNAL FLUORESCENCE AND INSTABILITY THRESHOLD}

We start by considering the effects on the quantum fluctuations in the PCOPO below threshold. On average the signal field vanishes everywhere $\left\langle\hat{A}_{1}(x)\right\rangle=0$ (or, equivalently, $\left\langle\hat{A}_{1}(k)\right\rangle=0$ in the far field) but, due to the nonlinearity of the medium, it is not in a coherent vacuum state either with or without the PC. As shown in Fig. 2(b), the mean number of photons in the far field is maximal at the critical wave number [17]. These large fluctuations (below threshold) in the

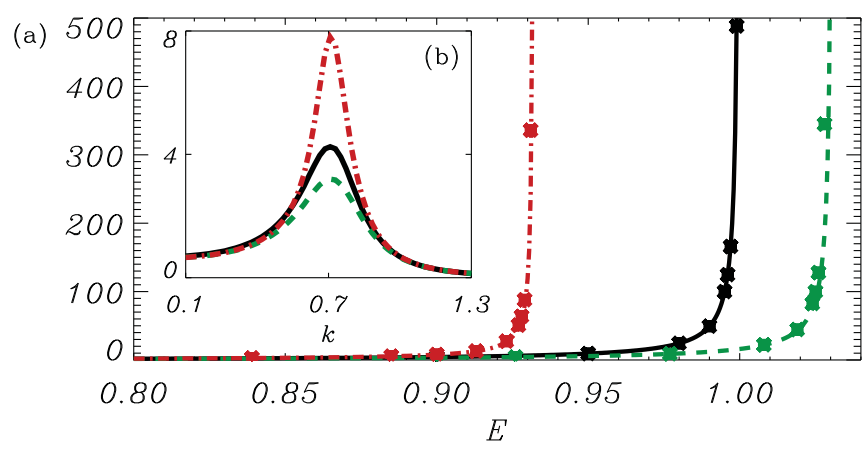

FIG. 2. (Color online) (a) Steady mean number of photons $\left\langle\hat{A}_{1}^{\dagger}\left(k_{c}\right) \hat{A}_{1}\left(k_{c}\right)\right\rangle$ increasing with the pump field $E$. The symbols are the results of numerical simulations of Eqs. (2) and (3) and the lines are obtained analytically within a linear approximation [28]. (b) $\left\langle\hat{A}_{1}^{\dagger}(k) \hat{A}_{1}(k)\right\rangle$ from numerical simulations for $E=0.9$, $\delta_{1}=-1\left(k_{c}=\sqrt{1 / 2}\right)$, and $\delta_{0}=0$. Black solid lines are for the OPO $\left(M_{0}=M_{1}=0\right)$, green dashed lines are for the PCOPO with $M_{0}=0$ and $M_{1}=0.5$ and the red dot-dashed lines are for the PCOPO with $M_{0}=0.5$ and $M_{1}=0$. All the represented quantities $E$, $k$, and $\left\langle\hat{A}_{1}^{\dagger}\left(k_{c}\right) \hat{A}_{1}\left(k_{c}\right)\right\rangle$ are dimensionless and scaled as in Ref. [18]. 
spatial modes of the pattern arising above threshold are known as noisy precursors and have been observed experimentally in different optical systems [26]. One motivation behind proposing PCOPOs is the intuition that this noise is expected to be inhibited by the PC band gap $[1,3]$ as actually found: The OPO $\left(M_{0}=M_{1}=0\right)$ exhibits a larger mean number of photons [Fig. 2(b), solid line] than the PCOPO with modulated $\Delta_{1}(x), M_{0}=0$ and $M_{1}=0.5$ [Fig. 2(b), green dashed line]. In contrast, it is rather surprising to find that the mean number of photons in the PCOPO can also be increased by the PC, as shown in Fig. 2 (red dot-dashed line, $M_{0}=0.5$ and $M_{1}=0$ ). In other words, the mean number of photons can either decrease or increase, with the same input energy, just by changing the amplitude modulation of the PC (i.e., $M_{0,1}$ ).

To understand why fluctuation emission can increase in spite of being in the PC band gap we need to take into account the presence of wave mixing between different frequencies in the parametric oscillator (which was not considered in Ref. [7]) and that the fluctuation strength is inherently related to the proximity to the instability threshold. In Fig. 2(a) fluctuations increase with the input $E$ approaching the instability point (vertical asymptotes, where the linear approximation used for analytical calculations breaks down). Consistently with the lower fluctuations in Fig. 2(b), we find that for the modulated signal detuning the threshold increases with respect to the OPO (as in the phenomenon of pattern inhibition with PCs [7,27]). However, modulation of the pump detuning leads to a lower instability threshold, so the PC actually favors the instability process.

We interpret these results by noticing that a signal detuning modulation $k_{\mathrm{PC}}=2 k_{c}$ is chosen to forbid the signal emission at $k_{c}$, which is in the PC band gap, and we actually find the expected result (dashed green lines in Fig. 2). However, when modulating the pump detuning with this periodicity, the PC actually changes the average pump profile too. In this case, due to the PC modulation of $\Delta_{0}$, the pump field (homogenous in an OPO below threshold) is no longer homogeneous, but spatially modulated. Moreover, the pump develops (below threshold) a pattern with the same periodicity $2 k_{c}$ of the OPO above threshold [25], favoring the pattern formation process in the signal at $k_{c}$. Therefore, for signal detuning modulation $k_{\mathrm{PC}}=2 k_{c}$ the signal emission at $k_{c}$ is in the band gap and the instability is inhibited, while if the pump detuning is modulated this actually excites the pump field at $2 k_{c}$, favoring the instability. In general, depending on the modulation strength of the signal and pump fields, the PC provides two competing mechanisms, inhibiting the signal spatial instability as in Refs. [7,27], but also imprinting in the pump the nonlinear structure favoring the instability process. A correspondent decrease or increase of the mean number of photons in the below-threshold signal is then found. Notably, if the PC modulates the pump detuning, the parametric threshold can be crossed for values even lower than in the case of a perfectly resonant $\mathrm{OPO}$, here $E=1$.

\section{SQUEEZING AND ENTANGLEMENT BELOW AND ABOVE THRESHOLD}

Apart from the strength of spatial fluctuations, an important aspect is the quantumness of the correlations. Nonclassical effects in multimode OPOs are known to exist between opposite far-field modes $+k$ and $-k$ due to the emission of photons pairs in the parametric down-conversion process [17]. In particular, two-mode squeezing is studied by considering the generic joint quadrature $\theta$,

$$
\Sigma_{\theta \phi}(k,-k)=\left[\hat{A}_{1}(k)+\lambda \hat{A}_{1}(-k) e^{i \phi}\right] e^{i \theta}+\text { H.c. },
$$

with $\phi$ the relative phase between the superposed spatial modes. Here we take $\lambda=1$.

Squeezing achieved below threshold increases with the pump intensity being maximum at the parametric threshold $[17,18]$. Due to the discussed PC effect on the parametric threshold, the squeezing attained in OPOs and PCOPOs will be compared at the same distance from the respective thresholds. Even if the PC significantly changes the intermode correlations leading to new nonvanishing terms with respect to the case of the OPO [in the PCOPO, for instance, $\left\langle\hat{A}_{1}^{2}(k)\right\rangle \neq 0$ ], we find [Fig. 3(a)] that squeezing achieves similar values in the OPO and in the PCOPO, the major difference being the dependence on the angles $\theta$ and $\phi$.

Important effects are found above threshold by considering squeezing between intense modes $\left[\left\langle\hat{A}_{1}\left( \pm k_{c}\right)\right\rangle \neq 0\right]$. In Fig. 3(b) the variance of $\Sigma_{\theta \phi}$ [Eq. (5)] for the OPO (black solid line) is compared with that for the PCOPO (dashed and dot-dashed lines). Even if the attained squeezing (minimum value of the plotted variance) is similar in all cases, there are important differences in the noise present in the unsqueezed quadrature (maximum value). Far from being in a minimum-uncertainty state, the OPO displays extremely large fluctuations in the unsqueezed quadrature [black solid line in Fig. 3(b)] due to the well-known phase diffusion between down-converted modes and to excess noise in their relative phases [23]. In spatially multimode devices, the visible effect is a diffusive motion of the pattern that has been related to translational symmetry breaking and noise excitation of the corresponding neutral Goldstone mode [29]. A major effect of the refractive index modulation of the PCOPO is that it is no longer translationally invariant: The formed pattern is locked in the position of the PC. This leads to a strong reduction of the unsqueezed quadrature variance in the PCOPO: A difference of two orders of magnitude is highlighted by the horizontal
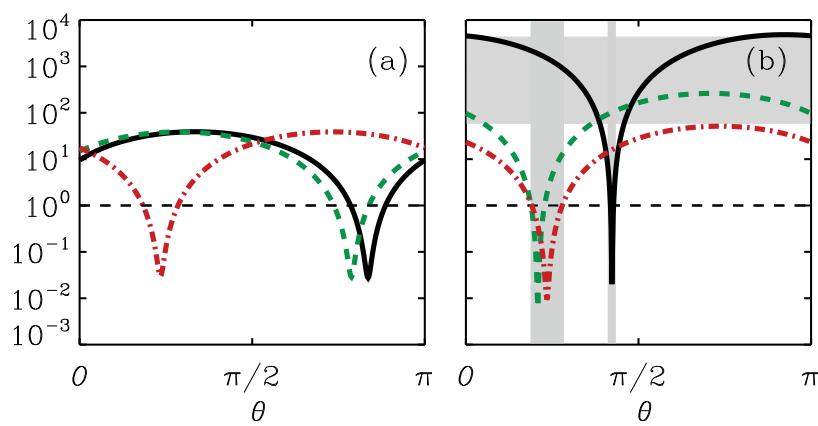

FIG. 3. (Color online) Variance of $\Sigma_{\theta \bar{\phi}}$ [from Eq. (5) with $\lambda=1$ ] as a function of the quadrature angle and for the superposition angle $\bar{\phi}$ giving the largest squeezing for each OPO and PCOPO configuration. The pump field is (a) $5 \%$ below threshold and (b) $2 \%$ above threshold. The horizontal dashed line is the shot noise and other lines are defined as in Fig. 2. Here and in the following figures, all correlations are dimensionless due to the scaling of the fields [18]. 
gray stripe in Fig. 3(b). An important consequence in view of applications is that the reduction of fluctuations in unsqueezed quadratures leads to a significant increase of the range of quadratures with sub-shot-noise fluctuations, as highlighted by the two vertical stripes in Fig. 3(b). A PCOPO is indeed more robust to changes in the choice of the local oscillator phase $\theta$ as more quadratures are actually squeezed.

Multimode OPOs allow one to generate not only squeezed but also spatially entangled states [17,30]. A series of key experiments recently reported spatial entanglement between light beams (a continuous variable regime) in different optical nonlinear devices [11]. Here we show how entanglement in parametric oscillators is changed by the presence of a PC, considering two well-known criteria [21,23]. One distinguishes states exhibiting the Einstein-Podolsky-Rosen (EPR) paradox [22] for conditional variances of position and momentum such that

$$
\mathcal{E}=\Delta^{2} \Sigma_{\theta_{0}, \phi_{0}} \Delta^{2} \Sigma_{\theta_{0}+\pi / 2, \phi_{0}+\pi} \leqslant 1
$$

for some choice of superposition and interference angles $\theta_{0}$ and $\phi_{0}$ [23]. Here the parameter $\lambda$ minimizes each variance of the joint quadrature Eq. (5). A second measure we consider is the inseparability condition

$$
\mathcal{I}=\Delta^{2} \tilde{\Sigma}_{\theta_{0}, \phi_{0}}+\Delta^{2} \tilde{\Sigma}_{\theta_{0}+\pi / 2, \phi_{0}+\pi} \leqslant 2\left(a^{2}+\frac{1}{a^{2}}\right),
$$

with $\tilde{\Sigma}_{\theta, \phi}=\left[a \hat{A}_{1}(k)+a^{-1} \hat{A}_{1}(-k) e^{i \phi}\right] e^{i \theta}+$ H.c. and the positive parameter $a$ [21]. Below threshold, OPOs and PCOPOs, both reach similar values of entanglement (for minima of $\mathcal{E}$ and $\mathcal{I}$ ) as well as squeezing. The most significant differences between OPOs and PCOPOs are found again above threshold (Figs. 4 and 5). The presence of the PC enhances quantum effects leading to a spatially entangled state. In this regime,
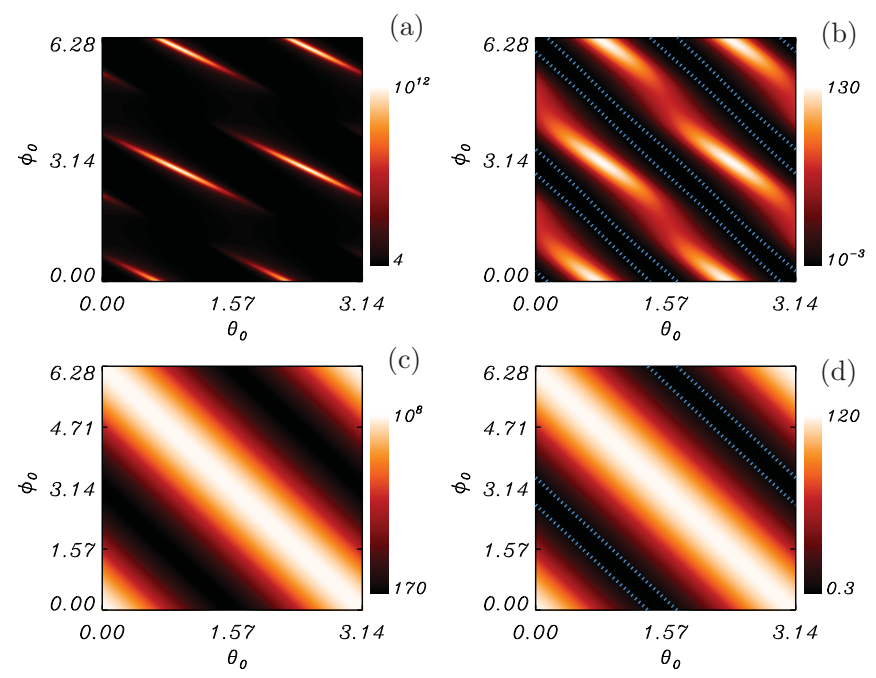

FIG. 4. (Color online) Variances of the (a) and (b) product $\mathcal{E}$ and (c) and (d) sum $\mathcal{I}$, as defined in Eqs. (6) and (7), for the output fields [31] of (a) and (c) OPOs and (b) and (d) PCOPOs when varying the superposition and interference angles $\theta_{0}$ and $\phi_{0}$. In (b) and (d) $M_{0}=0.5$ and $M_{1}=0$ (like for the red dot-dashed line in Figs. 2 and 3) and all results are for a pump $2 \%$ above threshold. Blue dotted lines limit regions for which Einstein-Podolsky-Rosen entanglement and inseparability are respectively predicted.

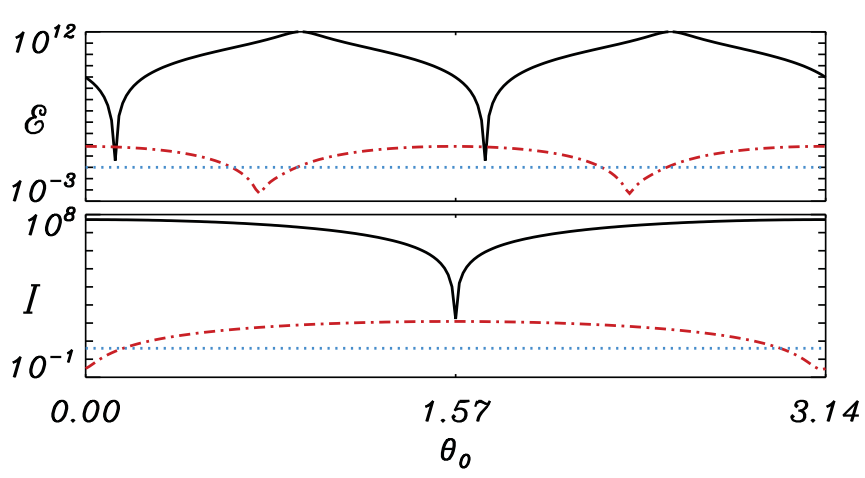

FIG. 5. (Color online) Product $\mathcal{E}$ and sum $\mathcal{I}$ of OPOs for the same parameters and normalization as in Figs. 4(a) and 4(c) (black solid lines) and of PCOPOs for the same parameters and normalization as in Figs. 4(b) and 4(d) (red dot-dashed lines), both $2 \%$ above threshold, plotted versus the quadrature angle for the superposition angle $\bar{\phi}_{0}$ that minimizes, respectively, $\mathcal{E}$ or $\mathcal{I}$. Entanglement is predicted below the blue dotted horizontal lines, as in Fig. 4 .

the mentioned phase diffusion leads to spikes at low frequency in noise spectra [23,29], preventing entanglement in the OPO, as we show in Figs. 4(a) and 4(c). In contrast, for a nontranslationally invariant system such as the PCOPO, we find significant regions in which both the EPR paradox Eq. (6) and state inseparability Eq. (7) are predicted. It is worth noting that in Fig. 4 the OPO and PCOPO are compared at the same distance from the instability threshold and that, even if the best performance is obtained when pump detuning is modulated (in Figs. 4(b), 4(d) and in Fig. 5 we considered $M_{0}=0.5$ and $M_{1}=0$ ) we find that all configurations of the PCOPO show some entanglement, which degrades when the $\mathrm{PC}$ is removed. We notice that for this parameter choice the PC improves the OPO performance, giving both a lower input energy threshold (Fig. 2) and entanglement (Fig. 5).

\section{CONCLUSIONS AND OUTLOOK}

As reported in the Introduction, several experiments have demonstrated the possibility of controlling both spontaneous emission and optical pattern formation by PCs. The latter is a complex phenomenon whose quantum aspects have been considered in several devices over the past two decades. In this work we have investigated the interplay between the nonlinear wave mixing and pattern formation taking place in OPOs and the periodic modulation imprinted by a PC, showing that it allows one to tune quantum fluctuations in different and unsuspected ways. We have considered as a prototypical transversally multimode device an OPO with an intracavity PC and we have shown that light emission for unstable modes in the band gap is not always prevented, as might be expected $[1,3,4]$. Competing mechanisms lead to either reduction or enhancement of quantum fluctuations for a fixed pump energy by changing the PC modulation strength. The possibility of controlling the mean number of photons just by modulating the refractive index is related to the increase [7,8] and decrease of the instability threshold. This light emission is characterized by a vanishing average field and is known in the (nonlinear classical optics) literature as a noisy precursor of a spatial modulation instability [26]. Looking 
at quantum effects such as squeezing and entanglement, the most important results are found above threshold where the breaking of the translational invariance due to the PC provides a strong mechanism to reduce (up to two orders of magnitude) the quadrature quantum fluctuations associated with spatial diffusion. This leads to squeezing over a significantly larger range of quadrature angles, thereby reducing the sensitivity of the choice of the phase of the local oscillator in squeezing measurements. Moreover, we show that the strong spatial locking due to the presence of the PC in the OPO allows one to generate inseparable as well as EPR entangled spatial beams.

Here we have shown results for PC modulations in an otherwise (transversally) translationally invariant system. Our conclusions remained unchanged when considering an injected beam that was not uniform but had a super Gaussian transverse profile. Further, numerical simulations of the stochastic equations (2) and (3) have been performed considering one transverse dimension, but similar results are expected when extending this analysis to two transverse dimensions for PCs modulated only in one direction (stripes) [28]. Open questions are the result of different transverse PC geometries (squares, hexagons, etc.) as well as different kinds of nonlinear cavities [16] when a PC is inserted. Another interesting possibility would be to induce near-field quantum correlations for strong modulations of the PC in the tight-binding limit. Our analysis of the interplay between nonlinearity and PC spatial modulation also might be relevant beyond light correlations in the context of polaritons and matter waves in periodic potentials [32].

\section{ACKNOWLEDGMENTS}

This work was supported by FISICOS (Grant No. FIS200760327), ECuSCo (Grant No. 200850I047), and CoQuSys (Grant No. 200450E566) projects and "Acció Especial" Grant No. AAEE0113/09. M.A.G.-M. acknowledges funding from the Fulbright Commission and FECYT.
[1] E. Yablonovitch, Phys. Rev. Lett. 58, 2059 (1987); S. John, ibid. 58, 2486 (1987).

[2] J. D. Joannopoulos, S. G. Johnson, J. N. Winn, and R. D. Meade, Photonic Crystals: Molding the Flow of Light, 2nd ed. (Princeton University Press, Princeton, 2008).

[3] S. Noda, M. Fujita, and T. Asano, Nature Photon. 1, 449 (2007), and references therein.

[4] P. Lodahl, A. F. van Driel, I. S. Nikolaev, A. Irman, K. Overgaag, D. Vanmaekelbergh, and W. L. Vos, Nature (London) 430, 654 (2004); D. Englund, D. Fattal, E. Waks, G. Solomon, B. Zhang, T. Nakaoka, Y. Arakawa, Y. Yamamoto, and J. Vuckovic, Phys. Rev. Lett. 95, 013904 (2005); M. Fujita, S. Takahashi, Y. Tanaka, T. Asano, and S. Noda, Science 308, 1296 (2005); T. LundHansen, S. Stobbe, B. Julsgaard, H. Thyrrestrup, T. Sünner, M. Kamp, A. Forchel, and P. Lodahl, Phys. Rev. Lett. 101, 113903 (2008).

[5] P. Lambropoulos, G. M. Nikolopoulos, T. R. Nielsen, and S. Bay, Rep. Prog. Phys. 63, 455 (2000).

[6] K. Busch, G. von Freymann, S. Linden, S. F. Mingaleev, L. Tkeshelashvili, and M. Wegener, Phys. Rep. 444, 101 (2007).

[7] D. Gomila, R. Zambrini, and G.-L. Oppo, Phys. Rev. Lett. 92, 253904 (2004).

[8] N. Marsal, D. Wolfersberger, M. Sciamanna, G. Montemezzani, and D. N. Neshev, Opt. Lett. 33, 2509 (2008); B. Terhalle, N. Radwell, P. Rose, C. Denz, and T. Ackemann, Appl. Phys. Lett. 93, 151114 (2008).

[9] M. I. Kolobov, Rev. Mod. Phys. 71, 1539 (1999).

[10] A. M. C. Dawes, L. Illing, S. M. Clark, and D. J. Gauthier, Science 308, 672 (2005).

[11] V. Boyer, A. M. Marino, R. C. Pooser, and P. D. Lett, Science 321, 544 (2008); A. M. Marino, R. C. Pooser, V. Boyer, and P. D. Lett, Nature (London) 457, 859 (2009); J. Janousek, K. Wagner, J. F. Morizur, N. Treps, P. K. Lam, C. C. Harb, and H.-A. Bachor, Nature Photon. 3, 399 (2009); G. Brida, M. Genovese, and I. R. Berchera, ibid. 4, 227 (2010).

[12] N. Treps, N. Grosse, W. P. Bowen, C. Fabre, H.-A. Bachor, and P. K. Lam, Science 301, 940 (2003).
[13] M. N. O’Sullivan Hale, I. A. Khan, R. W. Boyd, and J. C. Howell, Phys. Rev. Lett. 94, 220501 (2005); M. Lassen, V. Delaubert, J. Janousek, K. Wagner, H.-A. Bachor, P. K. Lam, N. Treps, P. Buchhave, C. Fabre, and C. C. Harb, ibid. 98, 083602 (2007).

[14] H. Vahlbruch, M. Mehmet, S. Chelkowski, B. Hage, A. Franzen, N. Lastzka, S. Gossler, K. Danzmann, and R. Schnabel, Phys. Rev. Lett. 100, 033602 (2008).

[15] Z. Y. Ou, S. F. Pereira, H. J. Kimble, and K. C. Peng, Phys. Rev. Lett. 68, 3663 (1992); A. S. Villar, L. S. Cruz, K. N. Cassemiro, M. Martinelli, and P. Nussenzveig, ibid. 95, 243603 (2005); A. S. Coelho, F. A. S. Barbosa, K. N. Cassemiro, A. S. Villar, M. Martinelli, and P. Nussenzveig, Science 326, 823 (2009).

[16] Quantum Imaging, edited by M. Kolobov (Springer, New York, 2007).

[17] A. Gatti, H. Wiedemann, L. A. Lugiato, I. Marzoli, G.-L. Oppo, and S. M. Barnett, Phys. Rev. A 56, 877 (1997); R. Zambrini, A. Gatti, L. Lugiato, and M. San Miguel, ibid. 68, 063809 (2003); I. Perez-Arjona, E. Roldan, and G. J. de Valcarcel, Europhys. Lett. 74, 247 (2006); M. Lassen, G. Leuchs, and U. L. Andersen, Phys. Rev. Lett. 102, 163602 (2009); B. Chalopin, F. Scazza, C. Fabre, and N. Treps, Phys. Rev. A 81, 061804(R) (2010).

[18] R. Zambrini, S. M. Barnett, P. Colet, and M. San Miguel, Eur. Phys. J. D 22, 461 (2003).

[19] L. A. Lugiato, M. Brambilla, and A. Gatti, Adv. At. Mol. Opt. Phys. 40, 229 (1999), Optical Pattern Formiation, edited by B. Bederson and $\mathrm{H}$. Walther.

[20] M. Peckus, K. Staliunas, Z. Nizauskaite, and V. Sirutkaitis, Opt. Lett. 32, 3014 (2007).

[21] L.-M. Duan, G. Giedke, J. I. Cirac, and P. Zoller, Phys. Rev. Lett. 84, 2722 (2000); R. Simon, ibid. 84, 2726 (2000).

[22] A. Einstein, B. Podolsky, and N. Rosen, Phys. Rev. 47, 777 (1935).

[23] M. D. Reid and P. D. Drummond, Phys. Rev. Lett. 60, 2731 (1988); Phys. Rev. A 40, 4493 (1989). 
[24] See Ref. 31 in M. Bache, P. Scotto, R. Zambrini, M. San Miguel, and M. Saffman, Phys. Rev. A 66, 013809 (2002) for the random number generator we refer to; R. Toral and A. Chakrabarti, Comput. Phys. Commun. 74, 327 (1993).

[25] G.-L. Oppo, M. Brambilla, and L. A. Lugiato, Phys. Rev. A 49, 2028 (1994).

[26] G. Agez, C. Szwaj, E. Louvergneaux, and P. Glorieux, Phys. Rev. A 66, 063805 (2002); N. Marsal, D. Wolfersberger, M. Sciamanna, and G. Montemezzani, ibid. 81, 031804(R) (2010).

[27] D. Gomila and G.-L. Oppo, Phys. Rev. E 72, 016614 (2005).
[28] M. A. Garcia March, M. M. de Castro, and R. Zambrini (unpublished).

[29] R. Zambrini, M. Hoyuelos, A. Gatti, P. Colet, L. Lugiato, and M. San Miguel, Phys. Rev. A 62, 063801 (2000).

[30] F. Castelli and L. A. Lugiato, J. Mod. Opt. 44, 765 (1997); A. Gatti, L. A. Lugiato, K. I. Petsas, and I. Marzoli, Europhys. Lett. 46, 461 (1999).

[31] M. J. Collett and C. W. Gardiner, Phys. Rev. A 30, 1386 (1984).

[32] A. V. Gorbach and D. V. Skryabin, Phys. Rev. B 82, 125313 (2010); A. Itah, H. Veksler, O. Lahav, A. Blumkin, C. Moreno, C. Gordon, and J. Steinhauer, Phys. Rev. Lett. 104, 113001 (2010); Yu. V. Bludov and V. V. Konotop, Phys. Rev. A 81, 013625 (2010). 